\title{
Particle Acceleration in the Sun and Beyond
}

\author{
Jeongwoo Lee († $^{* \dagger}$ \\ Seoul National University \\ E-mail: leejeastro.snu.ac.kr
}

This article reviews recent studies on cosmic rays originating in the heliosphere with emphasis on the role played by solar flares. In the first part, the physical drivers for high-energy particle acceleration in the heliosphere including solar flares, coronal mass ejections (CMEs), corotating interaction regions (CIR) and solar wind termination shocks are briefly discussed. We then introduce solar particle acceleration mechanisms in which shocks, turbulence, Alfvén waves, and magnetic reconnection respectively play a role. It is demonstrated that the properties of accelerated particles in the Sun are known in considerable detail because magnetic reconnection geometry is visible through electromagnetic radiations, and imaging spectroscopy at hard X-ray and radio wavelengths is available. In the second part, we attempt to relate our knowledge of solar flare particles to several research topics on solar energetic particles (SEPs). The topics include: (1) possible influence of solar magnetic field structure upon the energy spectra and time profiles of the resulting SEPs, (2) temporal, spatial and spectral properties of SEP electrons measured in interplanetary space in comparison with those remotely observed in the Sun, and (3) the energydependent onset time of SEP protons and its implication on the proton acceleration beyond the Sun. It is argued that we should take advantage of solar imaging spectroscopy at X-ray and radio wavelengths to complement the cosmic ray studies largely based on in-situ observations in order to disentangle the roles played by solar flares and CMEs in accelerating SEPs and better understand the relationship between solar flares and SEPs.

35th International Cosmic Ray Conference - ICRC2017

10-20 July, 2017

Bexco, Busan, Korea

* Speaker.

${ }^{\dagger}$ Thanks the organizers of this conference and the KOFST for the support under the Brainpool program. 


\section{Introduction}

This article is intended to introduce recent studies on the heliospheric particle acceleration with emphasis on the role of solar flares. It is now known that the particle acceleration occurs not only on the Sun but at various sites throughout the heliosphere. Accordingly, diverse drivers have been identified including not only solar flares, but Coronal Mass Ejections (CME), Corotating Interaction Regions (CIR) and solar wind termination shocks. Some of the accelerated particles with energy ranging from a few tens of $\mathrm{keV}$ to $\mathrm{GeV}$ are identified to come from the Sun and called Solar Energetic Particles (SEPs). SEPs may originate either from a solar-flare site or shock waves associated with CMEs.

Often cited in many reviews of SEPs is the solar flare myth (Gosling 1993), that all energetic particles are accelerated in the solar flares. The myth started with the first observation of highenergy particles from the Sun as sudden increases in intensity in ground-level ion chambers during the large solar events in 1942 (Forbush, 1946) and dominated in the SEP community over 40 years until turned out to be wrong. Shift of this paradigm to include the role of CMEs led to a significant progress in understanding the particle acceleration in solar particle events (SPEs).

In this article, we focus on the particle acceleration during solar flares and transport of them out into the space to appear as SEPs. We emphasize the importance of investigating the properties of SEPs at the time of escaping the Sun as seed population for more energetic cosmic rays. Such information is also valuable as reference for the further acceleration of SEPs in the interplanetary (IP) space. For the background and context of the article, we here briefly review the physical drivers for particle acceleration in the heliosphere.

\subsection{Solar flares}

Solar flares are the most powerful explosions in the solar system, and also capable of energizing a large number of charged particles to relativistic speeds. Significant progress has been gained in our understanding of solar flares. There are two areas in which remote sensing observation of solar flares can have advantage over direct in-situ observations. First, imaging spectroscopy at X-ray wavelengths has been available with the Reuven Ramaty High Energy Solar Spectroscopic Imager (RHESSI) (Hurford et al. 2002). RHESSI's high spectral resolution together with spatial information (angular and spectral resolutions down to 2.3 arcsec and $1 \mathrm{keV}$, respectively, over a wide energy range) promoted various particle acceleration models. The achievement includes more complicated magnetic field geometries and topologies (e.g., Petrosian et al. 2002, Battaglia and Benz 2006) in addition to the earlier discovery of loop-top hard X-ray source (Masuda et al. 1994). Second, the location and temporal evolution of the magnetic reconnection geometry is quite visible, which is important for exploring solar particle acceleration and escaping from the Sun associated with magnetic reconnection (Kocharov et al. 2005, 2007). Now is the best time for such research since many space-borne solar observations from, for instance, Helios, Ulysses, RHESSI and STEREO missions are available.

\subsection{CMEs}

CME is the main competitor to the solar flares in producing the SEPs. Kahler et al., (1986, 1987a) found a high correlation between SEP events and CMEs. However, that correlation alone 
does not tell us that SEP acceleration occurs at the CME-driven shock and not at the reconnection region behind the CME. It is therefore of interest how to separate these two components in a given event. A simple criterion has been that impulsive time profiles are due to solar flares and gradual time profiles implies CMEs. This simple criterion may not work because not all solar flare sources are weaker, and sometimes, comparatively strong. Various aspects of gradual and impulsive SEP events have been compared and described in a variety of review articles (Reames 1995, 1997, Kahler 1992, 1994, Gosling 1993, Cliver 1996). If a solar flare is the only source, we should be able to trace back to the solar source along the spiral magnetic field lines. However, the presence of shocks ahead of CMEs can make the SEP distribution broader. In the major (gradual) SEP events, the particle injection from the Sun is a prolonged process, so that the entire time profile of the SEP source should be considered (Klein et al. 2008). CMEs may also occur in succession to create an even more extended source.

\subsection{CIRs}

CIR is a region of plasma and field compression that is formed by fast solar wind overtaking slow wind ahead owing to the effect of corotation with the Sun (Smith and Wolfe 1976), and the most plausible acceleration sites are traveling interplanetary shocks, where the relative speed between the shocks and particles is sufficiently low that the particles encounter the shocks many times (Giacalone et al. 1997). The boundaries of CIRs are representative of such shock waves. As the CIR propagates approximately beyond Earth's orbit, its boundaries are usually steepened by the forward and reverse shocks. Interplanetary observations have revealed energetic particle events in association with the CIR (e.g., Barnes and Simpson 1976, Tsurutani et al. 1982, Desai et al. 1999). Observations by the Ulysses spacecraft identified pickup ions (Fisk et al. 1974) acceleration in the CIR, where stochastic acceleration due to transit-time damping of magnetic turbulence is proposed as the initial process (Schwadron et al. 1996).

\subsection{Solar wind termination shocks}

Solar wind termination shocks are considered to be responsible for accelerating anomalous cosmic rays (ACRs). ACRs were discovered in the early 1970s as a new component of cosmic rays when unexpected enhancements were observed in the energy spectra of 1 to $50 \mathrm{keV} / \mathrm{nuc} \mathrm{He}, \mathrm{N}$, and O measured in interplanetary space. After the discovery, Fisk et al. (1974) proposed that ACRs represent a sample of neutral interstellar particles that have drifted into the heliosphere, become ionized by the solar wind or UV radiation, and then accelerated to energies of tens of MeV/nuc in the outer heliosphere. (Klecker 1995). In 1977-78, diffusive shock acceleration was introduced, and subsequently developed in detail, providing compelling explanations for, e.g., the observed acceleration in co-rotating interaction regions, and a likely explanation for the acceleration of ACRs at the termination shock of the solar wind. These observations led to the development of a new acceleration mechanism, the pump acceleration mechanism (Fisk and Gloeckler 2014), which can account for the observed uncommon spectral index (Krimigis et al. 1997).

\subsection{Scope of this paper}

The goal of this review is to demonstrate that the particle acceleration during solar flares are studied in considerable detail, and argue that we should be able to exploit such knowledge in the 
studies of heliospheric cosmic rays. Accordingly, CIR and ACR as well as acceleration with the planetary magnetospheres are not included in this paper. For a more comprehensive review of particle acceleration in the heliosphere, we refer to Balogh et al. (2012). Plan of this paper is as follows: solar flare acceleration mechanisms are selectively reviewed in $\$ 2$. We then discuss ejection of solar flare particles out to the space with emphasis on the role of magnetic fields ( $\$ 3)$, properties of the SEP electrons relative to solar flares (\$4), and a new classification of SEP protons (§5). A brief concluding remark is given in $§ 6$.

\section{Acceleration of Solar Particles}

Particle acceleration during solar flares cannot be viewed as a subset of galactic cosmic ray acceleration. First of all, the diffusive shock acceleration, the major mechanism invoked for astrophysical systems, is not particulary effective during solar flares. Solar particle acceleration is more closely associated with magnetic reconnection in one way or another, since the primary source of the energy is in stressed, current-carrying magnetic fields and released via magnetic reconnection. One of the advantages of solar flare study is that many features of magnetic reconnection such as the magnetic cusp, flare ribbon motion and their direction relative to local magnetic fields are visible through electromagnetic observations. In solar physics community, the consensus is established for a theoretical framework that explains such observations of the eruptive flares (Forbes et al. 2006). This so-called standard solar flare model also explains the gradual phase of a flare as the reconnection and closing down of the field opened in a transient manner by the associated CME, which is of interest to the research of SEPs. Since then, more realistic and complex geometries for solar magnetic reconnection have been proposed. Turbulence generated by magnetic reconnection is also extensively studied for stochastic acceleration of particles during solar flares. Instead of extensive survey of all possible models for solar particle acceleration, we selectively review mechanisms that are considered to be more relevant to SEPs. For more comprehensive reviews of this subject, we refer to Aschwanden (2002) and Zharkova et al. (2011).

\subsection{Particle acceleration by shocks}

Shock acceleration is the most frequently invoked mechanism in astrophysical plasma context, ever since a very simple model of shock wave acceleration predicts a power-law spectrum of the accelerated particles in good agreement with observations of galactic cosmic rays (Blandford and Ostriker 1978, Bell 1978, Cargill 1991, Giacalone and Jokipii 1996). However, this simple model, though very elegant, has a number of shortcomings. Inclusion of Coulomb at low energies and synchrotron at high energies or the influence of accelerated particles on the shock structure (Amato and Blasi 2005) can cause significant deviations from a simple power law. Moreover, shocks are unable to accelerate low energy background particles efficiently and shock acceleration requires the injection of fairly high-energy seed particles. Also, shock acceleration requires some scattering agents (most likely plasma waves or turbulence) to cause repeated passage of the particles through the shock front. In the case of quasi-parallel shocks, in which the magnetic field is nearly parallel to the normal of the shock front, the rate of energy gain is governed by the pitch-angle diffusion rate. Although there may be some indirect morphological evidence for the existence of such shocks in flares (Sui and Holman 2003), there is little direct evidence for the occurrence of these kinds of 
shocks near the top of flaring loop, where the acceleration seems to be taking place during the impulsive phase. Furthermore, such shocks typically do not appear in 3-D MHD simulations of the reconnection process. Many of the features that make acceleration of cosmic rays by shocks attractive are thus not directly applicable to the solar flare environment.

\subsection{Stochastic acceleration by turbulence}

Stochastic acceleration is a main contender for the particle acceleration mechanism in flares, as is capable of explaining the the rate, number and complicated spectral morphology inferred from solar flare observations. The most widely studied drivers for this type acceleration are plasma wave turbulence (Hamilton and Petrosian 1992, Pryadko and Petrosian 1997) and cascading MHD turbulence (Miller et al. 1996). In the first scenario, a presupposed level of plasma wave turbulence in the ambient plasma accelerates ambient particles of energy stochastically. It has been shown that for flare conditions, plasma-wave turbulence can accelerate the background particles to the required high energies within the very short timescale derived from hard X-ray observations (e.g., Hamilton and Petrosian 1992, Miller et al. 1996). More importantly, at low energies, and especially in strongly magnetized plasmas, the acceleration rate of the ambient electrons may significantly exceed the scattering rate for particular pitch angles and directions of propagation of this turbulence (Pryadko and Petrosian 1997, 1998), leading to an efficient acceleration. Miller et al. (1996) applied cascading fast-mode waves to the stochastic acceleration of electrons in impulsive flares, providing a consistent model for electrons and ions, except for ${ }^{3} \mathrm{He}$ which is not selectively enhanced by cascading waves. An extensive review of the status of various theories of particle acceleration in impulsive flares has been published recently (Miller et al. 1997). Until very recently it was thought that electrons above $\sim 20 \mathrm{keV}$ contained much more energy than protons above $\sim 1$ $\mathrm{MeV}$. Recently, however, it has become clear from gamma-ray line measurements that the energy content in accelerated electrons and ions is comparable (Ramaty et al. 1996), 10 $0^{31}-10^{32}$ ergs in large flares.

\subsection{Acceleration by Alfvénic turbulence}

Large-scale Alfvén waves are often invoked for particle acceleration in space. As motivated by in situ observations and theories for energy transport and electron acceleration in the Earth's magnetosphere during sub-storms (Stasiewicz et al. 2000), it is suggested that the energy is transported through the corona not by particle beams but by Alfvén-wave pulses, launched in the primary reconnection site and carrying a large Poynting flux towards the chromosphere Fletcher and Hudson (2008). The presence of such pulses has recently been verified in MHD simulations by Birn et al. (2009). An ideal, incompressible, non-linear Alfvén mode traveling in a completely uniform plasma does not steepen into a shock, or dissipate due to Landau damping (Barnes and Hollweg 1974), but the presence of density fluctuations, including those generated by the wave itself, can lead it to decay via a parametric instability into a forward propagating ion-acoustic wave and a backward-propagating Alfvén wave (e.g. Goldstein 1978). Because the Alfvén speed in such an environment is so high (higher than the electron thermal speed, such that an average electron lags behind the wave), the details of the wave propagation require two-fluid or even full kinetic simulations. Such simulations performed in the case of the magnetosphere (Kletzing 1994) indicate that 
a parallel electric field is generated by the wave pulse, in which ambient electrons can be accelerated to twice the Alfvén speed. Similar considerations for solar coronal conditions indicated that modest non-thermal energies of a few tens of $\mathrm{keV}$ could be achieved in this manner. McClements and Fletcher (2009) have confirmed this for solar plasma in the test-particle limit.

\subsection{Acceleration via magnetic reconnection}

Solar magnetic reconnection may occur in association with large-scale magnetic structure and possible magnetic field geometries involved with magnetic reconnection include 3-D reconnecting current sheets, magnetic fan and spine structure, collapsing current sheets among others. Particle acceleration in 3-D reconnecting current sheets has been studied through MHD simulations of particle acceleration rates although the number of particles that can be accelerated in a current sheet is limited by the volume encompassed by the current sheet (Holman 1985, Litvinenko 1996). Magnetic fan and spine structures are also important as they can have a 3-D magnetic null and thus reconnection and particle acceleration are possible (Lau and Finn 1990, Priest and Titov 1996). Spine reconnection in 3-D MHD reconnection simulations can naturally produce two symmetric jets of energetic particles escaping along the spine, while the fan reconnection produces azimuthally localized ribbons of high energy particles in the fan plane. Both features, in principle, are often observed in flares, e.g., magnetic jets and two-ribbon flares as discussed in Fletcher et al. (2011). Jet-like structure can also be important in ejecting solar energetic particles into space.

Collapsing current sheets are also regarded as an important setting for particle acceleration in the Sun in which In addition, betatron acceleration, possibly coupled with first-order Fermi acceleration in collapsing current sheets, can account for the electron number and energies in the form of collapsing magnetic traps. Somov and Kosugi (1997), Karlický and Kosugi (2004) and Bogachev and Somov $(2005,2007)$ have carried out simulations of the acceleration of electrons in collapsing magnetic traps. Although not a macroscopic instability, Furth et al. (1963) demonstrated that tearing-mode instabilities can generate during reconnection in a current sheet. Accordingly, large current sheets are often observed in solar flares (Sui and Holman 2003).

\subsection{Acceleration by termination shock}

A termination shock is often invoked in the standard model of solar flares (e.g. Forbes 1986). Due to the strong curvature of the magnetic field lines in the vicinity of the diffusion region in a helmet-type reconnection, the slowly inflowing plasma shoots away from the reconnection site as a hot jet. These oppositely-directed jets are embedded between a pair of slow magnetosonic shocks. If the speed of this jet is super-Alfvénic, a fast magnetosonic shock (a termination shock) can be established. The appearance of such shocks was predicted in the numerical simulations of Forbes (1986), Shibata et al. (1995) and Somov and Kosugi (1997). Radio measurements (Aurass et al. 2002, White et al. 2011) of an X17.2 flare reveal the termination shock as a standing radio source. A strong enhancement of the electromagnetic emission in the hard X- and gamma-ray range up to $10 \mathrm{MeV}$ is simultaneously observed with the appearance of the radio signatures of the shock (Aurass and Mann 2004). Chen et al. (2015) reported observations of a solar flare termination shock and trace its dynamics using high-cadence radio imaging spectroscopy, which suggests the role of a termination shock in accelerating energetic electrons in solar flares. These observations indicate that the termination shock could well be the source of the high-energy electrons emitting 
the hard X-rays and gamma-rays. A fast magnetosonic shock formed during the MHD simulations of a reconnection process is accompanied by a compression of both the magnetic field and the density. Thus, it represents a moving magnetic mirror at which charged particles can be reflected and accelerated through a process termed shock-drift acceleration, which is capable of accelerating electrons up to $10 \mathrm{MeV}$ (Mann et al. 2006).

\section{Solar Cosmic Rays: Role of Magnetic Field}

3-D magnetic field structure of a solar flare source must be an important factor for understanding the property of SEPs originating from that source, because charged particles accelerated during solar flares will interact with ambient magnetic fields until they escape from the Sun. Direction of local magnetic field with respect to the shock propagation will also determine the nature of shock acceleration. It is therefore worthwhile to study 3-D magnetic fields of solar flare sources for predicting the lives of SEPs afterwards. In spite of such potential importance, the role of magnetic field in the transport of accelerated particles out to space has not received much attention from both communities. Solar physicists concern themselves with the problem of how accelerated particles are trapped in magnetic loops or precipitate down to interact with the lower solar atmosphere, while cosmic ray physicists are more interested in energetic particles detected near the Earth. It is yet to be investigated whether or not the precipitating and escaping populations are accelerated by the same mechanism. In this section we highlight a few works which addressed this issue by exploring the role of 3-D magnetic structure on the particles escaping from the Sun.

\subsection{Energy dependence of SEPs}

If macroscopic structure of magnetic field has influence on the particle acceleration or, at least, that of escaping particles, we may be able to determine, from the active region structure, the phase space density of solar cosmic rays. Kocharov et al. (2012) addressed this problem using a numerical model of coronal shock acceleration. They used a semicircular magnetic field line and discussed how the shock geometry affect the resulting spectrum. The model calculation shows that when the shock geometry in a particular magnetic tube changes from nearly parallel to perpendicular, the resulting SEP spectrum at the top of a transequatorial loop, resembles a wide beam, which is very different from the standard power-law spectrum in a steady state. It is also noted that the shock-accelerated particles may escape from more than one coronal location, and reaccelerated via stochastic process, and the magnetic tube expansion can make the SEP spectra even more complicated. The model could explain the observed proton energy spectra, ion and electron timing, and abundances in the beginning of major solar energetic particle (SEP) events. This result indicates that seed spectrum of the SEP will vary depending on the ejection geometry, and that nonstandard energy spectra of the shock-accelerated ions may result depending on the magnetic environment of the solar corona.

\subsection{Time profiles of SEPs}

It also worths investigating whether time profiles of ejected particles, and thus the SEP time profiles afterward have relation with active region structure, . Kocharov et al. (2017) addressed this question by studying two similar events of extreme solar particle events: the 1998 May 2 event 
(GLE 56) and the 2003 November 2 event (GLE 67). The main idea is to check if two sources regions with identical or similar magnetic structure result in similar SPEs. The 1998 May 2 event is associated with a flare and a coronal mass ejection (CME), which was well observed by the Nancay Radioheliograph. The 2003 November 2 event the CME liftoff in the low corona was observed by the Mauna Loa Solar Observatory (MLSO). Those complementary data sets are analyzed jointly with the broadband dynamic radio spectra, EUV images, and other data available for both events. As a result, a common scenario is found for both eruptions, including the flare's dual impulsive phase, the CME-launch-associated decimetric-continuum burst, and the late, low-frequency type III radio bursts at the time of the relativistic proton injection into the interplanetary medium. The analysis supports the idea that the two considered events start with emission of relativistic protons previously accelerated during the flare and CME launch, then trapped in large-scale magnetic loops and later released by the expanding CME. Moreover, apparently similar GLEs from the similar active region implies that once the source structure is well understood, we may predict the GLE time profiles, and maybe its strength too.

\section{Solar Cosmic Rays: Electrons}

Electrons are the best observable species among solar flare particles, as they produce ample electromagnetic radiation at hard X-rays and radio wavelengths via nonthermal bremsstrahlung and gyrosynchrotron mechanisms, respectively. These radiations carry unique information on the acceleration, trapping and escape of energetic electrons. They also emit coherent radiation such as solar radio bursts. Especially type III bursts have been used for characterization of electron beams as they leave their acceleration site in the low corona toward interplanetary space. It is highly desirable to exploit such electron diagnostics for interpreting of the properties of SEPs. For distinction from protons of SEPs, let us hereafter use the term, SEP electrons.

\subsection{Timing analysis of solar solar energetic electrons}

Studies of SEP electrons analysis the delayed timing between the HXRs and the solar release of escaping electrons have been made utilizing the time-shifting analysis (TSA) and the velocity dispersion analysis (VDA) with type III bursts as reference (Krucker et al. 1999, Haggerty and Roelof 2002). If these delayed events are indeed accelerated later in the event by shocks, no correlation between the HXR photon spectrum and the in-situ observed electron spectrum is expect to be found. Haggerty and Roelof (2002) studied time profiles of scatter-free impulsive beamlike electron events (38-315 keV) measured at 1 AU to identify 79 such impulsive events during 19972000. Detailed statistical analysis of the timing between the electron injection and the soft Xray, microwave, chromospheric $\mathrm{H} \alpha$, and metric and decametric type III radio emission shows that the near-relativistic electrons measured by the Advanced Composition Explorer/Electron, Proton, and Alpha Monitor in orbit around the Earth's first Lagrangian point are associated with western hemisphere events and are injected with a median delay of $\sim 10$ minutes after the start of the electromagnetic emissions. The delayed injection and a weak statistical correlation between the intensities of the near-relativistic electrons and the characteristics of the electromagnetic emissions, indicates that the escaping near-relativistic electron populations are not directly related to those that generate the prompt flare-related emissions. The observations are consistent with acceleration of 
the escaping near-relativistic electrons by an outgoing fast coronal shock launched near the time of the prompt electromagnetic emissions.

\subsection{Spectral analysis of solar energetic electrons}

Although studies using RHESSI's spectroscopy significantly advanced our knowledge on solar flare electrons, relatively fewer studies were made for the electrons escaping from the Sun. Krucker et al. (2007) carried out such a study by combining RHESSI X-ray observations with in-situ observations of energetic electrons from the WIND spacecraft. Statistical results of 16 events with a close temporal agreement between the HXR and the in-situ detected electrons (taking the time of flight of the escaping electrons into account) show a correlation between the HXR photon spectral index and the electron spectral index observed in-situ thus indicating a common acceleration mechanism. Furthermore, the solar X-ray source structure of these events look similar showing hot loops with HXR footpoints plus an additional HXR source separated from the loop. This source structure can be explained by a simple magnetic reconnection model with newly emerging flux tubes that reconnect with previously open field lines, so-called interchange reconnection. Xie et al. (2016) also performed a study of such energy-dependence of SEP onset times with distinction of electrons and protons for the events from December 2006 to March 2014. They derived solar particle release (SPR) times for the 0.25-10.4 MeV electrons using the TSA and the proton SPR times using both the TSA and the VDA to find that near-relativistic electrons and high-energy protons are released at the same time within 8 min for most (16 of 23) SEP events and that for 7 low-intensity events of the 23 , large delays occurred for $6.5 \mathrm{MeV}$ electrons and $90.5 \mathrm{MeV}$ protons relative to $0.5 \mathrm{MeV}$ electrons. Whether these delays are due to times needed for the evolving shock to be strengthened or due to particle transport effects remains unsolved. Such characterization of electron beams as they leave their acceleration site in the low corona toward interplanetary space must be an ideal tool to be used in future. However, its success largely depends on the sensitivity limit of X-ray instrument as worked out by Saint-Hilaire et al. (2011).

\subsection{Imaging of solar energetic electrons}

Spatial distribution of accelerated electrons in the Sun can be imaged through their nonthermal X-ray bremsstrahlung emission. Such investigation is now possible with the RHESSI's imaging spectroscopy. For instance, Liu et al. (2008) analyzed RHESSI observation of flare ribbon-like hard X-ray (HXR) sources up to $100 \mathrm{keV}$ found in the 2005 May 13 M8.0 flare to determine, along a flare ribbon, an anticorrelation relationship between the local HXR flux and the local HXR spectral index, and also an anticorrelation between the HXR spectral index and the local electric field along the ribbon, which suggests electron acceleration by the electric field during flares. Of interest to comic ray study is imaging spectroscopy of high-energy electrons escaping from the Sun to space. Chen et al. (2013) performed decimetric observations of type III radio bursts using the new technique of radio dynamic imaging spectroscopy provided by the recently upgraded Karl G. Jansky Very Large Array. which can be signatures of suprathermal electron beams propagating in the low corona below $15 \mathrm{Mm}$. The detailed structure of the magnetic release site revealed by their observation indicate that the localized energy release is highly fragmentary in time and space, supporting a bursty reconnection model for particle acceleration. 
Imaging of energetic electrons around CMEs would be more interesting, but had been considered not possible due to weaker intensity as expected. Bastain et al. (2001) performed direct imaging of the expanding CME loops at radio wavelengths using the Nancay radioheliograph. This so-called radio CME was nearly simultaneously with the onset of an associated type II radio burst, shock-accelerated type III radio bursts. Radio spectra are constructed as a function of position inside the CME loop, as the imaging was made at four frequencies. The spectral analysis reveals that it results from nonthermal synchrotron emission from electrons with energies of $\sim 0.5-5 \mathrm{MeV}$ interacting with magnetic fields of $\sim 0.1$ to a few gauss. This challenging observation was possible because those energetic electrons are trapped in the relatively strong magnetic fields inside the CME emit via synchrotron radiation. Otherwise no hard X-ray imaging is available at such heights $\geq 3 R_{\odot}$. It should also be noted that this observation does not show the particle acceleration in the shock front ahead of CME. Observed are probably associated with the flare-accelerated electrons which are injected into the CME loop. More radio imaging of CMEs is needed to better understand particle acceleration around CMEs.

\section{Solar Cosmic Rays: Protons}

Protons are the main component for SEPs, and their temporal and energy-dependent properties in the interplanetary space are well measured through in-situ observations. One difference from SEP electrons is that solar counterpart to SEP protons is hardly observed through electromagnetic radiations. For spatial and spectra information of protons just escaping from the Sun, we should reply on theoretical models which can be relate the proton acceleration to electron acceleration in the Sun. Whether the observed SEP protons are due to solar flares or CMEs is still an issue, and the simple rule that an impulsive (gradual) time profile indicates its association with solar flares (CMEs) can be an insufficient argument. A few recent studies that characterize SEPs according to their energy-dependent onset timing may be useful in this regard.

\subsection{SEP onset time and flux enhancement pattern}

Kim et al. (2014) have selected 42 strong SPEs observed by the SOlar and Heliospheric Observatory (SOHO)/Energetic and Relativistic Nuclei and Electron (ERNE) with the criterion of incoming energetic $(>10 \mathrm{MeV})$ protons exceeding 10 particle flux unit (pfu) at geosynchronous satellite altitudes, together with information of associated flares, CMEs, and IP type II radio bursts during 1997-2012 from the list published by the National Oceanic and Atmospheric Administration/Space Weather Prediction Center ${ }^{1}$. Investigation of the temporal pattern of flux enhancement reveals four different groups of the SPEs reveals that all events can be classified into four distinct groups called A to D. The SEPs in group A show the flux enhancement by the largest amount $\left(\sim 10^{4}\right)$ among others, especially in the low and middle energy channels. Those in group B show the flux enhancement by the smallest amount in overall. Especially they exhibit almost no enhancement with time in the lowest energy channels. In contrast, the SEPs in group C show relatively large flux enhancements at low energy channels and least enhancements at higher energy channels. Finally, the SEPs in group D behave similar to group A, but with modest flux enhancements (increase

\footnotetext{
${ }^{1}$ http://www.swpc.noaa.gov/ftpdir/indices/SPE.txt
} 
by a factor of $10^{2}$ ) in all channels. In summary, all flare-related events (31\%) show a single pattern of the acceleration starting from the lower energy channel, whereas the CME-related events show three different acceleration patterns: the proton accelerations starting from either lower or higher energy channels or both.

\subsection{Timing of SEPs relative to type III bursts}

Kim et al. (2015) extended the SEP classification by examining how these SEP groups A-D are associated with the related flare phenomena based on the closeness of their onset times. The SEPs in the group A tend to precede flares, CME, and type II radio burst, while the SEPs in the group $\mathrm{C}$ tend to follow others. Only the SEPs in group A have onset times closer to those of flares than the CME's first appearance time. In groups B-D, SEP onset times are closer to the CME appearance times. The SEPs in group B show relatively longer time gaps between the CME and type II radio burst, which implies the source location of type II radio burst in the low corona. The SEPs in group $C$ have very delayed onsets with respect to CMEs and type II radio bursts. The SEPs in group D show relatively short time differences of each phenomenon. As a criterion for how fast the accelerations are processing in respective energy channels, the time differences between onsets in the highest and lowest energy channels, $\Delta t=t_{H}-t_{L}$ is used. In A, the proton acceleration starts from the lowest energy and quickly comes up at high energies with $\Delta t \approx 17 \mathrm{~min}$ on average. In $\mathrm{B}$, the proton acceleration starts in the higher energy and enhances later in the lower energy with $\Delta t \approx 26$ min. $\mathrm{C}$ shows the acceleration pattern similar to that of A but with $\Delta t \approx 35 \mathrm{~min}$, which is much longer than that of group $\mathrm{A}$. In $\mathrm{D}$ a very short time difference, $\Delta t \approx-1.8 \mathrm{~min}$, is found. This classification scheme is more obvious when we look at the temporal flux changes in the multichannel energy spectrum. The flare-associated SEPs (A) tend to show a large flux enhancement by a factor of $\sim 10^{4}$ in a short time, while the CME-associated SEPs (B-D) tend to show relatively a weak flux enhancement by a factor of $\sim 10^{2}$. In short, the energy-dependent onset times provide a simple way to discriminate the flare-associated SEPs from CME-associated SEPs.

\subsection{Implication of the energy-dependent onset time}

The above classification of SPEs recovers the conventional notion of impulsive vs. gradual events in that the rapid flux increase seen in the flare-associated SPEs (group A) resembles the property of impulsive SPEs, and the relatively weak and slow flux enhancements in the CMEassociated SPEs (groups B-D) resembles that of the gradual SPEs, respectively. However, the energy-dependent pattern of flux enhancement gives another piece of information that the proton acceleration mechanism operating in CMEs (B-D) may differ from that in solar flares (A). Compared with group $\mathrm{C}$, the acceleration seems more efficient at high energies in group $\mathrm{B}$, for which the diffusive shock acceleration mechanism (Zank et al. 2000) may be more appropriate. Group C can then be explicable by another acceleration mechanism that is more effective at low energies with a limiting high energy such as acceleration by DC electric field. The apparent similarity in the spectral variation between groups A and D may imply a common acceleration mechanism operating in both flares and CMEs but different acceleration efficiency. For instance, the proton acceleration model developed for solar flares (Miller et al. 1990) predicts the acceleration efficiency to be related to the energy density in either magnetosonic or Alfven waves, in which case, the difference between group A and D may thus be due to the lower wave energy density in CMEs than in flares. 
Relating to the interplanetary medium conditions, a recent model that has been discussed heavily is the twin-CME model (Ding et al., 2013). It was also suggested that the large SPE with preceding CMEs may not be due primarily to CME interaction, but a general increase of both background seed particles and more frequent CMEs during times of higher solar activity (Kahler and Vourlidas 2014). To conclude, flare-associated SEPs retain the acceleration pattern of solar flares, whereas CME-associated SEPs exhibit a variety of energy-dependent behaviors not found in solar flares.

\section{Concluding Remarks}

In this review, we have introduced the recent progress in understanding the particle acceleration during solar flares and attempted to put the knowledge in the context of SEPs. As noted elsewhere, solar research has an advantage in that we can perform imaging spectroscopy of flares at hard X-rays and radio wavelengths to determine the temporal, spatial, and energy-dependent properties of high-energy flare electrons, which can be compared with those of SEPs measured in IP space. This is the case for SEP electrons. For SEP protons, however, no observable solar counterpart is available, and theoretical models can be used to relate those protons to the electrons accelerated during solar flares. Another advantage is that spatial structure of the magnetic energy release is accessible to some extent, which can also help exploring the particles escaping from the Sun. Specifically we can derive information on global magnetic field structures for the escaping particles, properties of SEPs inside coronal magnetic traps and CME loops, type III radio bursts as evidence for the magnetic reconnection and time-dependent particle injection from the Sun.

The present selection of the topics in solar flare particle acceleration and its relation to SEPs is by no means complete. For simplicity, we have not discussed the pitch-angle dependent transport from the Sun to the Earth and the chemical abundance of SEPs, both of which serve as critical constraints in cosmic ray studies. We would like to emphasize that with ever increasing capability of solar instruments, the spatial structure of the source region in the Sun and its interaction with escaping particles should be more accessible in future. It is in this area of imaging spectroscopy of solar flares and CMEs that we may expect a rapid progress in the heliospheric cosmic ray study beyond the traditional research tools can offer. Solar flare particles themselves are less energetic, but important as seed particles for SEPs. The data and theoretical ideas developed for solar flares should therefore be exploited to understand how strong SEPs occur.

\section{Acknowledgments}

This work was supported in part by the 2017 Brainpool Program of the KOFST. I also thank the Korea Astronomy and Space Science Institute for the R\&D program, Development of a Solar Coronagraph on International Space Station (Project No. 2017-1-851-00), supervised by the Ministry of Science, ICT, and Future Planning.

\section{References}

[1] Amato, E., Blasi, P. 2005 A general solution to non-linear particle acceleration at non-relativistic shock waves MNRAS 364 L76 
[2] Aschwanden, M. J. 2002 Particle acceleration and kinematics in solar flares - A Synthesis of Recent Observations and Theoretical Concepts (Invited Review) SSRv 1011

[3] Aurass, H., Vrnak, B., Mann, G. 2002 Shock-excited radio burst from reconnection outflow jet? A\&A 384273

[4] Aurass, H., Mann, G. 2004 Radio Observation of Electron Acceleration at Solar Flare Reconnection Outflow Termination Shocks ApJ 615526

[5] Balogh, A., Bykov, A., Lin, R., Raymond, J., Scholer, M. 2012 Cosmic Plasmas and Particle Acceleration: An Introduction SSRv 1731

[6] Barnes, A., Hollweg, J. V. 1974 Large-amplitude hydromagnetic waves JGR 792302

[7] Bastian, T. S., Pick, M., Kerdraon, A., Maia, D., Vourlidas, A. 2001 The Coronal Mass Ejection of 1998 April 20: Direct Imaging at Radio Wavelengths ApJ 558L 65

[8] Battaglia, M., Benz, A. O. 2006 Relations between concurrent hard X-ray sources in solar flares A\&A 456751

[9] Bell, A. R. 1978 The acceleration of cosmic rays in shock fronts MNRAS 182147

[10] Birn, J., Fletcher, L., Hesse, M., et al. 2009 Energy Release and Transfer in Solar Flares: Simulations of Three-Dimensional Reconnection ApJ 6951151

[11] Blandford, R. D., Ostriker, J. P. 1978 Particle acceleration by astrophysical shocks ApJ 211 L29

[12] Bogachev, S. A., Somov, B. V. 2005 Comparison of the Fermi and Betatron Acceleration Efficiencies in Collapsing Magnetic Traps AstL 31537

[13] Bogachev, S. A., Somov, B. V. 2007 Formation of power-law electron spectra in collapsing magnetic traps AstL 3354

[14] Brosius, J. W. and Stephen M. 2006 Radio Measurements of the Height of Strong Coronal Magnetic Fields Above Sunspots at the Solar Limb ApJ 641 L69

[15] Cargill, P. J. 1991 The Interaction of Collisionless Shocks in Astrophysical Plasmas ApJ 376771

[16] Chen, B., Bastian, T. S., White, S. M., Gary, D. E., Perley, R., Rupen, M., Carlson, B. 2013 Tracing Electron Beams in the Sun's Corona with Radio Dynamic Imaging Spectroscopy, ApJ 763L 21

[17] Chen, B., Bastian, T. S., Shen, C., Gary, D. E., Krucker, S. Glesener, L. 2015 Particle acceleration by a solar flare termination shock, Science 3501238

[18] Cliver, E. W. 1996 Solar flare gamma-ray emission and energetic particles in space AIPC 37445

[19] Ding, L., Jiang, Y., Zhao, L., Li, G. 2013 The "Twin-CME" Scenario and Large Solar Energetic Particle Events in Solar Cycle 23 ApJ 76330

[20] Fisk, L. A., Kozlovsky, B., Ramaty, R. 1974 An interpretation of the observed oxygen and nitrogen enhancements in low-energy cosmic rays ApJ. 190 L35

[21] Fisk, L. A., and Gloeckler, G. 2014 The case for a common spectrum of particles accelerated in the heliopshere: Observations and theory JGRA 1198733

[22] Fletcher, L., Hudson, H. S. 2008 Impulsive Phase Flare Energy Transport by Large-Scale Alfven Waves and the Electron Acceleration Problem ApJ 6751645

[23] Fletcher, L., Dennis, B. R., Hudson, H. S., Krucker, S., Phillips, K., Veronig, A., Battaglia, M., Bone, L., Caspi, A., Chen, Q., Gallagher, P., Grigis, P. T., Ji, H., Liu, W., Milligan, R. O., Temmer, M. 2011 An Observational Overview of Solar Flares SSRv 15919 
[24] Forbes, T. G. 1986 Fast-Shock Formation in Line-tied Magnetic Reconnection Models of Solar Flares ApJ 305553

[25] Forbes, T. G. Linker, J.A. Chen, J., et al., 2006 CME Theory and Models, Space Science Reviews 123,251

[26] Forbush, S. E. 1946 Three Unusual Cosmic-Ray Increases Possibly Due to Charged Particles from the Sun PhRv 70771

[27] Furth, H. P., Killeen, J., Rosenbluth, M. N. 1963 Finite-Resistivity Instabilities of a Sheet Pinch PhFl 6459

[28] Giacalone, J., Jokipii, J. R.1996 Perpendicular transport in shock acceleration JGR 10111095

[29] Giacalone, J., Jokipii, J. R., Decker, R. B., Krimigis, S. M., Scholer, M., Kucharek, H. 1997 Preacceleration of Anomalous Cosmic Rays in the Inner Heliosphere ApJ 486471

[30] Goldstein, M. L. 1978 An instability of finite amplitude circularly polarized Afven waves ApJ 219700

[31] Gosling, J. T. 1993 The Solar Flare Myth JGR 9818937

[32] Haggerty, D. K., Roelof, E. C. Impulsive Near-relativistic Solar Electron Events: Delayed Injection with Respect to Solar Electromagnetic Emission 2002 ApJ 579841

[33] Hamilton, R. J., Petrosian, V. 1992 Stochastic Acceleration of Electrons. I. Effects of Collisions in Solar Flares ApJ 398350

[34] Holman, G. D. 1985 Acceleration of runaway electrons and Joule heating in solar flares ApJ 293584

[35] Hurford, G. J., Schmahl, E. J., Schwartz, R. A. et al. 2002 The RHESSI Imaging Concept SoPh 21061

[36] Kahler, S. W., Cliver, E. W., Cane, H. V., et al. 1986 Solar filament eruptions and energetic particle events ApJ 302504

[37] Kahler, S. W., Cliver, E. W., Cane, H. V., et al. 1987 Solar Energetic Proton Events and Coronal Mass Ejections Near Solar Minimum ICRC 3121

[38] Kahler, S. W. 1992 Solar flares and coronal mass ejections ARA\&A 30113

[39] Kahler, S. W. 1994 Injection profiles of solar energetic particles as functions of coronal mass ejection heights ApJ 428837

[40] Kahler, S. W., Vourlidas, A. 2014 Do Interacting Coronal Mass Ejections Play a Role in Solar Energetic Particle Events? ApJ 78447

[41] Karlicky, M., Kosugi, T. 2004 Acceleration and heating processes in a collapsing magnetic trap A\&A 4191159

[42] Kim, R.-S., Cho, K.-S., J. Lee, J., Bong, S.-C., Park, Y.-D. 2014 A refined classification of SPEs based on the multienergy channel observations JGRA 1199419

[43] Kim, R.-S., Cho, K.-S., Lee, J., Bong, S.-C., Joshi, A. D., Park, Y.-D. 2015 Characteristics of four SPE groups with different origins and acceleration processes JGRA 1207083

[44] Klecker, B. 1995 The Anomalous Component of Cosmic Rays in the 3-D Heliosphere SSRv 72419

[45] Klein, K.-L., Krucker, S., Lointier, G., Kerdraon, A. 2008 Open magnetic flux tubes in the corona and the transport of solar energetic particles A\&A 486589

[46] Kletzing, C. A. 1994 Electron acceleration by kinetic Alfvén waves JGR 9911095 
[47] Kocharov, L., Lytova, M., Vainio, R., Laitinen, T., Torsti, J. 2005 Modeling the Shock Aftermath Source of Energetic Particles in the Solar Corona ApJ 6201052

[48] Kocharov, L., Saloniemi, O., Torsti, J. et al. 2007 High-Energy Protons Associated with Liftoff of a Coronal Mass Ejection ApJ 659780

[49] Kocharov, L., Vainio, R., Pomoell, J., Valtonen, E., Klassen, A., Young, C. A. 2012 Non-standard Energy Spectra of Shock-accelerated Solar Particles ApJ 75387

[50] Kocharov, L., Pohjolainen, S., Mishev, A. et al. 2017 Investigating the Origins of Two Extreme Solar Particle Events: Proton Source Profile and Associated Electromagnetic Emissions ApJ 83979

[51] Krimigis, S. M., Bostrom, C.O., Armstrong, T.P. et al. 1997 The Low Energy Charged Particle / LECP / experiment on the Voyager spacecraft SSRv 21329

[52] Krucker, S., Larson, D. E., Lin, R. P., Thompson, B. J. 1999 On the Origin of Impulsive Electron Events Observed at 1 AU ApJ 519864

[53] Krucker, S., Christe, S., Glesener, L. et al. 2014 First Images from the Focusing Optics X-Ray Solar Imager ApJ 793 L32

[54] Krucker, S., Kontar, E. P., Christe, S., Lin, R. P. 2007 Solar Flare Electron Spectra at the Sun and near the Earth ApJ 663L 109

[55] Krucker, S., Kontar, E. P., Christe, S., Glesener, L., Lin, R. P. 2011 Electron Acceleration Associated with Solar Jets ApJ 74282

[56] Lau, Y.-T., Finn, J. M. 1990 Three-dimensional Kinematic Reconnection in the Presence of Field Nulls and Closed Field Lines ApJ 350672

[57] Litvinenko, Y. E. 1996 A New Model for the Distribution of Flare Energies SoPh 167321

[58] Liu, C., Lee, J., Jing, J., Gary, D. E., Wang, H. 2008 The Spatial Distribution of the Hard X-Ray Spectral Index and the Local Magnetic Reconnection Rate ApJ 672L 69

[59] Mann, G., Aurass, H., Warmuth, A. 2006 Electron acceleration by the reconnection outflow shock during solar flares A\&A 454969

[60] Masuda, S., Kosugi, T., Hara, H., Tsuneta, S., Ogawara, Y. 1994 A loop-top hard X-ray source in a compact solar flare as evidence for magnetic reconnection Nature 371495

[61] McClements, K. G., Fletcher, L. 2009 Inertial AlfvÃl'n Wave Acceleration of Solar Flare Electrons ApJ 6931494

[62] Miller, J. A., Guessoum, N., Ramaty, R. 1990 Stochastic Fermi acceleration in solar flares ApJ 361 701

[63] Miller, J. A., Larosa, T. N., Moore, R. L. 1996 Stochastic Electron Acceleration by Cascading Fast Mode Waves in Impulsive Solar Flares ApJ 461445

[64] Miller, J. A., Cargill, P. J., Emslie, A. G., et al. 1997 Critical issues for understanding particle acceleration in impulsive solar flares JGR 102 14631M

[65] Petrosian, V., Donaghy, T. Q., McTiernan, J. M. 2002 Loop Top Hard X-Ray Emission in Solar Flares: Images and Statistics ApJ 56945

[66] Priest, E. R., Titov, V. S. 1996 Magnetic Reconnection at Three-Dimensional Null Points RSPSA 354 2951 
[67] Pryadko, J. M., Petrosian, V. 1997 Stochastic Acceleration of Low-Energy Electrons in Cold Plasmas ApJ 482774

[68] Pryadko, J. M., Petrosian, V. 1998 Stochastic Acceleration of Low-Energy Electrons in Plasmas with Finite Temperature ApJ 495377

[69] Ramaty, R., Mandzhavidze, N., Hua, X. 1996 High Energy Solar Physics AIPC 374

[70] Reames, Donald V. 1995 Solar energetic particles: A paradigm shift RvGeS 33585

[71] Reames, D. V., Kahler, S. W., Ng, C. K. 1997 Solar Energetic-Particle Spectra and the Structure of Coronal Mass Ejections ICRC 1185

[72] Saint-Hilaire, P., Krucker, S., Christe, S., Lin, R. P. 2011 The X-ray Detectability of Electron Beams Escaping from the Sun ApJ 696941

[73] Schwadron, N. A., Fisk, L. A., Gloeckler, G. 1996 Statistical acceleration of interstellar pick-up ions in co-rotating interaction regions GeoRL 232871

[74] Shibata, K., Masuda, S., Shimojo, M., et al. 1995 Hot-Plasma Ejections Associated with Compact-Loop Solar Flares ApJ 451 L83

[75] Smith, E. J., Wolfe, J. H. 1976 Observations of interaction regions and corotating shocks between one and five AU: Pioneers 10 and 11 GeoRL 2137

[76] Somov, B. V., Kosugi, T. 1997 Collisionless Reconnection and High-Energy Particle Acceleration in Solar Flares ApJ 485859

[77] Stasiewicz, K., Bellan, P., Chaston, C., et al. 2000 Small Scale Alfvenic Structure in the Aurora SSRv 92423

[78] Sui, L., Holman, G. D. 2003 Evidence for the Formation of a Large-Scale Current Sheet in a Solar Flare ApJ 596 L251

[79] White, S. M., Bellan, P., Chaston, C., et al. 2011 The Relationship Between Solar Radio and Hard X-ray Emission SSRv 159225

[80] Xie, H., Makela, P., Gopalswamy, N., St. Cyr, O.C. 2016 Energy dependence of SEP electron and proton onset times, 2016 JGRA 1216168

[81] Zank, G. P., Rice, W. K. M., Wu, C. C. 2000 Particle acceleration and coronal mass ejection driven shocks: A theoretical model JGR 10525079

[82] Zharkova, V. V., Arzner, K., Benz, A. O. et al. 2011 Recent Advances in Understanding Particle Acceleration Processes in Solar Flares SSRv 159357 